UDC 347.195 .21

LBC 67.404

\title{
THE PUBLICITY OF THE MODERN RUSSIAN STATE AND THE PROTECTION OF INDIVIDUAL RIGHTS (CERTAIN QUESTIONS)
}

\author{
Yanina Ya. Keil \\ Volgograd Institute of Management - the Branch of the Russian Presidential Academy of National Economy \\ and Public Administration under the President of the Russian Federation, Volgograd, Russian Federation
}

\section{Victoriya A. Usanova}

Volgograd Institute of Management - the Branch of the Russian Presidential Academy of National Economy and Public Administration under the President of the Russian Federation, Volgograd, Russian Federation

Introduction: there are quite a lot of works devoted to the research of publicity and transparency of the functioning of the modern Russian state, however, this area is multi aspect and multifaceted, so many questions remain understudied. So far, providing the publicity of the legal bodies' activities by the online publishing of the judicial decisions is facing the problems of protecting the information rights of the citizens. The purpose of the study is to reveal some aspects of protection of the rights of the individual while providing the transparency of the functioning of the Russian state on the Internet. Methods: there have been applied in aggregate the methods of scientific knowledge, including the basic methods, such as the formal and legal method, the methods of systemacity, analysis and synthesis. Results: it is proved that the current system of posting the judicial and other acts on an open source website often violates the human rights and requires the improvements both in the law enforcement practice and the current legislation. Conclusions: the law enforcement practice of publishing the judicial decisions does not meet the requirements of the legislation. The public authorities and local governments implement the law on providing the transparency of their functioning more broadly than it is provided.

Keywords: transparency, judicial act, publicity, regulation, human rights, information security.

УДК 347.195.21

ББК 67.404

\section{ТРАНСПАРЕНТНОСТЬ СОВРЕМЕННОГО РОССИЙСКОГО ГОСУДАРСТВА И ЗАЩИТА ПРАВ ЛИЧНОСТИ (НЕКОТОРЫЕ ВОПРОСЫ)}

\author{
Янина Яковлевна Кайль \\ Волгоградский институт управления - филиал Российской академии народного хозяйства \\ и государственной службы при Президенте РФ, г. Волгоград, Российская Федерация \\ Виктория Александровна Усанова \\ Волгоградский институт управления - филиал Российской академии народного хозяйства \\ и государственной службы при Президенте РФ, г. Волгоград, Российская Федерация
}

Введение: исследованию открытости и гласности функционирования современного российского государства посвящено достаточно много работ, однако данная сфера многоаспектная и многогранная, поэтому большинство вопросов остаются малоизученными. Обеспечение открытости деятельности судебных органов власти путем размещения решений судов в сети Интернет на сегодняшний день сталкивается с проблемами защиты информационных прав граждан. Цель исследования - раскрыть некоторые аспекты защиты прав личности при обеспечении открытости функционирования российского государства в сети Интернет. Методы: применены в совокупности методы научного познания, среди которых основные - формально-юридический, системный, анализ и синтез. Результаты: обосновано, что действующая система от- 


\title{
ТЕОРИЯ И ПРАКТИКА ГОСУДАРСТВЕННО-ПРАВОВОГО РАЗВИТИЯ
}

крытости размещения судебных и иных актов зачастую нарушает права человека и требует совершенствования как правоприменительной практики, так и действующего законодательства. Выводы: правоприменительная практика размещения решений судов не соответствует требованиям законодательства. Органы государственной власти и местного самоуправления реализуют законодательство об обеспечении открытости их функционирования более широко, чем это предусмотрено.

Ключевые слова: транспарентность, судебный акт, открытость, гласность, нормативный правовой акт, права человека, информационная безопасность.

INSERM 347.195.21

BCB 67.404

\section{LA TRANSPARENCE DE L'ETAT RUSSE MODERNE ET LA PROTECTION DES DROITS DE LA PERSONNE HUMAINE (CERTAINES QUESTIONS)}

\author{
Janina Ya. Keil \\ Institut de l'Administration Publique de Volgograd - filiale de l'Académie Russe de l'Economie Nationale \\ et de l'Administration Publique, Volgograd, Russie \\ Victoria A. Usanova \\ Institut de l'Administration Publique de Volgograd - filiale de l'Académie Russe de l'Economie Nationale \\ et de l'Administration Publique, Volgograd, Russie
}

Introduction: beaucoup de recherches sont consacrées à l'étude de l'ouverture et de la transparence du fonctionnement de l'état russe moderne. Cependant ce domaine est multidimensionnel et compliqué; de nombreuses questions légales sont encore mal examinées. Le mécanisme de la transparence du système judiciaire appliqué à présent en Russie quand les décisions des tribunaux sont placées sur Internet est aujourd'hui confronté à la nécessité dans de la protection des droits d'information des citoyens. Le but de la recherche est de révéler certains aspects de la protection des droits individuels, tout en assurant la transparence du fonctionnement de l'état russe à l'aide de l'Internet. Les méthodes utilisées: nous avons utilisé un ensemble intégré des méthodes de recherche y compris la méthode légale formelle, la méthode systématique, l'analyse et la synthèse. Les résultats: il est prouvé que le système actuel de la mise en ligne des décisions des tribunaux, des actes judiciaires et d'autres documents contenant l'information personnelle violent les droits humains et doit être révisé en tant que le mécanisme juridique utilisé dans la législation en vigueur russe. La conclusion: la pratique de l'application de la loi quand les décisions des tribunaux sont entièrement mises en ligne ne répond pas aux exigences de la législation russe. Ainsi les autorités de l'État et les collectivités locales misent en œuvre la législation garantissant la transparence de leur fonctionnement plus largement qu'il est justifié et prévu.

Les mots-clés: la transparence, les décisions des tribunaux, l'ouverture, l'expression ouverte, la réglementation, les droits de la personne humaine, la sécurité de l'information.

\section{Introduction}

L'informatisation caractérise l'état actuel du développement de la société russe, à savoir il y a lieu la mise au point et la mise en œuvre actives des technologies de l'information, des méthodes et des systèmes d'information dans tous les domaines d'activités sociales et publiques. L'informatisation globalement joue un rôle positif et crucial dans le développement de l'humanité. Mais en outre de l'impact positif de la sensibilisation de la société et de la transparence $\mathrm{du}$ fonctionnement de l'état, il existe des problèmes avec la protection des droits des citoyens lors de la mise en ligne publique de telles informations.

Il y a de nombreuses menaces à la sécurité de l'information personnelle; tout d'abord, il existe la nécessité de protéger les citoyens contre l'accès injustifiéà leurs données personnelles. Deuxièmement, la protection des personnes humaines contre l'affichage des informations sur les différents sites d'Internet, y compris sur les sites officiels des pouvoirs publics et des autonomies locales. Cette pratique non seulement contribue à la mise en œuvre des droits individuels, mais dans certaines situations, empêchent leur réalisation, en particulier dans le cas du droit à l'effort créateur, etc. 
La transparence du fonctionnement de l'état russe ne doit pas interférer avec et porter atteinte aux droits et aux intérêts légitimes des individus, de la société, de l'État, ni de leur taux de protection. En particulier, la stratégie de sécurité nationale, approuvé par le décret présidentiel du 31 décembre 2015 sous le numéro 683, définit la "sécurité nationale de la Fédération de Russie comme un état de sécurité de l'individu, de la société et de l'État contre les menaces internes et externes, qui fournissent la réalisation des droits et libertés constitutionnelles des citoyens russes, la digne qualité et le niveau de vie, la souveraineté, l'indépendance, l'intégrité nationnale et territoriale, le développement économique et social durable de la Fédération de Russie" [4]. La sécurité nationale comprend non seulement la défense du pays, mais aussi d'autres types de sécurité prévues par la législation russe et la Constitution de la Fédération de Russie. Il s'agit par exemple de la sécurité nationale, dans le domaine de l'information, de l'écologie, de l'économique, du transport, de la sécurité énergétique et de la sécurité sociale de l'individu.

\section{La protection des droits individuels lors de la mise en ligne des décisions judiciaires}

La protection de l'information personnelle peut être considérée comme une condition particulière de l'existance de la personne humaine, lorsque celle-ci est libre de toute intervention influençant son environnement informationnel. Les intérêts de l'individu dans la sphère de l'information consistent à la réalisation des droits constitutionnels de l'homme et du citoyen à l'accès à l'information pour la mise en œuvre d'activités non interdites par le droit pour le développement physique, spirituel et intellectuel, ainsi que pour la protection de l'information assurant la sécurité personnelle [2].

Nous sommes d'accord avec le V.P. Belyaev, G.S. Belyaeva, S.Y. Chapchikov que "la sécurité (en toutes ses formes y compris la sécurité nationale, économique, environnementale, d'information, démographiques, etc. ...) est l'une des principales catégories de la science et de la pratique juridique moderne" [1, p. 45].

Conformément aux dispositions de la partie 1 de l'article 123 de la Constitution de la
Fédération de Russie, les procédures des tribunaux judiciaires sont ouvertes [3]. L'expression ouverte prévoit le principe démocratique de la justice appuyant sur l'accès libre à et sur la capabilité de discuter l'information rendue publique, des audiences et des actes judiciaires [7, p. 187]. Conformément à l'article 15 de la Loi Fédérale du 22 décembre 2008 numéro 262-FZ "Sur l'accès à l'information sur l'activité des tribunaux de la Fédération de Russie" (dénommé ci-après la loi numéro 262-FZ), la mise en œuvre du principe constitutionnel de la transparence et de l'expression ouverte de la procédure judiciaire prévoit entre outre le devoir de la mise en ligne des actes judiciaires [6].

En règle générale, tout citoyen, indépendamment du fait s'il est ou il n'est pas un participant à l'instance, a le droit de se familiariser avec les décisions, les arrêts et les verdicts du tribunal adoptés lors des procédures judiciaires ouvertes.

D'une part, les citoyens ont le droit d'être tenus informés de l'état d'avancement de l'examen des cas, ainsi que d'avoir l'accès aux actes judiciaires publics placés par le biais d'un réseau (Internet, en particulier). D'autre part, l'état doit protéger les citoyens dont les données personnelles sont présentes dans de tels actes. Ainsi la question se pose: comment assurer l'ouverture et la transparence de la procédure judiciaire, sans perturber la sécurité de l'information personnelle?

Les actes judiciaires peuvent contenir les informations variées relatives à la personne humaine, des secrets d'Etat, commerciaux, banquaires, d'assurance et d'autres données confidentielles. Conformément à la clause "g" de la partie 2 de l'article 14 de la loi numéro 262-FZ, la mise en ligne des décisions judiciaires est obligatoire. L'article 15 de la loi numéro 262-FZ spécifie l'ordre de la mise en ligne des actes judiciaires soit dans leur intégralité soit sous une forme tronquée, selon les informations qui y sont contenues. La loi numéro 262-FZ établit une soidisant dépersonnalisation des décisions judiciaires des tribunaux de juridiction générale lorsqu'elles sont mises sur Internet, c'est à dire les données personnelles en sont exclues.

Après avoir examiné quelques textes des actes judiciaires des tribunaux de compétence générale russe placés sur Internet, il est possible 
de conclure qu'il existe la non-conformité à grande échelle avec les exigences légales. D'une part, il y a une violation du droit à l'information des citoyens parce que l'information nécessaire est trop souvent enlevée de façon déraisonnable des textes des actes judiciaires. Par exemple, il est supprimé de mentionner les noms des participants à l'essai, les dates des événements, ainsi toutes les données qui permettent de les identifier sont exclues des actes juridiques. Très souvent, les tribunaux retirent les numéros de référence, les dates de l'adjudication, les caractéristiques quantitatives des objets d'empiétement criminel, des détails des délits figurant dans des actions judiciaires. En conformité avec la législation russe en vigueur, les tribunaux peuvent supprimer d'autres informations disponibles sur internet même si elles ne sont pas directement liées aux données personnelles. D'autre part, les responsables du rendement disponibles à l'accès sur un réseau des textes des actes judiciaires, violent des droits des participants à l'instance, lorsque certaines informations auraient du être supprimées des actes judiciaires, à cause de leur accessibilité à tous les citoyens. Ainsi, les droits des participants sont violés dans les tribunaux de première instance de compétence générale.

\section{La protection des droits individuels lors de la mise en ligne de l'information sur les activités des pouvoirs publics et des autonomies locales}

Il est nécessaire d'examiner un autre aspect de la société de l'information, à savoir l'obligation des organismes publics et des autorités locales d'informer les citoyens sur leurs activités sur leurs sites Web. L'article 13 de la loi fédérale du 9 février 2009 numéro 8-FZ "Sur l'accès à l'information sur les activités des organes publiques et des collectivités locales" (dénommé ci-après la loi numéro 8-FZ) [5] définit la liste principale d'information obligatoirement mise sur les sites internet officiels des pouvoirs publics russes. Les renseignements publics comprennent l'information sur les fonctions normatives telles que les actes juridiques normatifs adoptés, les textes des projets de lois et d'autres actes juridiques normatifs et les réglementations municipals, ainsi que les règlements administratifs et les normes de services de l'état et des municipalités.

Le fonctionnement du site des organes publics doit être organisé de manière à assurer un accès libre et sans entrave à ces données. Conformément à la loi numéro 8-FZ la liste d'informations qui doivent obligatoirement être rendues disponibles rur Internet reste encore ouverte. La législation actuelle ne prévoit pas explicitement que le cadre juridique de cet organe doivent être disponible en ligne, mais les collectives publiques peuvent facultativement le faire. De nombreuses autorités publiques guidées par de bonnes intentions, affichent sur leurs sites Web un large éventail des actes juridiques, ceuxci ne sont pas directement liés à leurs activités ou profil.

Cette obligation du rendement des renseignements ouverts conduit au fait que les experts qui analysent les règlements d'activité des organes, y compris ceux qui sont affichés sur les sites officiels des pouvoirs publics et des autonomies locales ont des difficultés de publier leurs conclusions et résultats d'analyse parce que leur recherche possède un niveau insuffusant de l'originalité du texte. Les systèmes actuels de la vérification des textes pour garantir leur authenticité trouvent le plagiat et des citations erronées dans les recherches en référant aux sites Internet des différents organes du pouvoir d'Etat et des autonomies locales et aux réglementations qui y sont présentées. L'imperfection et le travail contingent de ces programmes antiplagiat, résulte en impossibilité de publier des résultats du travail analytique des experts à cause des exigences formelles de l'authenticité de la publication, en particulier à cause de l'insuffisance de l'originalité du texte d'auteur. Conséquemment l'exigence de la transparence du fonctionnement des pouvoirs publics de l'état russe, et de ses organes publics conduit à une restriction du droit au travail et à la recherche scientifique.

\section{Conclusion}

Ainsi, pour protéger les droits des individus, les autorités publiques et les gouvernements locaux, en collaboration avec les institutions de la société civile, doivent améliorer la protection des droits de la personne humaine et de ses libertés à travers du développement des systèmes législatif, judiciaire et du maintien de l'ordre. 


\section{REFERENCES}

1. Belyaev V.P., Belyaeva G.S., Chapchikov S.Yu. Evolyutsiya ponyatiya natsionalnoy bezopasnosti (istoriko-pravovoy srez) [Evolution of the Concept of National Security (Historical and Legal Viewpoint)]. Vestnik Volgogradskogo gosudarstvennogo universiteta. Seriya 5, Yurisprudentsiya [Science Journal of Volgograd State University. Jurisprudence], 2015, no. 3 (32), pp. 45-53.

2. Gubanov V.M., Solomin V.P., Mikhaylov L.A. Chrezvychaynye situatsii sotsialnogo kharaktera $i$ zashchita ot nikh [Emergent Cases of Social Character and Their Prevention]. Available at: http://www. nnre.ru/yurisprudencija/chrezvychainye_situacii_ socialnogo haraktera i zashita ot nih/p12.php. (accessed December 7, 2016).

3. Konstitutsiya Rossiyskoy Federatsii: (prinyata vsenarodnym golosovaniem 12.12.1993) (s uchetom popravok, vnesennykh zakonami RF o popravkakh $k$ Konstitutsii RF ot 30.12.2008 № 6FKZ, ot 30.12.2008 № 7-FKZ, om 05.02.2014 № 2FKZ, ot 21.07.2014 № 11-FKZ) [Constitution of the Russian Federation: (Adopted by the All-People Voting on December 12, 1993) (taking into account amendments made through Russian laws on amendments to Constitution of the Russian Federation of December 30, 2008 no. 6-FKL; of December 30, 2008 no. 7-FKL, of February 5, 2014 no. 2-FKL, of July 21, 2014 no. 11- FKL]. Access from Reference Legal System "KonsultantPlyus".

4. Ukaz Prezidenta $R F \ll O$ Strategii natsionalnoy bezopasnosti Rossiyskoy Federatsii» ot 31.12.2015 № 683 [Decree of President of the Russian Federation "On the Strategy of National Security of the Russian Federation" of December 31, 2015 no. 683]. Access from Reference Legal System "KonsultantPlyus".

5. Federalnyy zakon «Ob obespechenii dostupa $k$ informatsii o deyatelnosti gosudarstvennykh organov $i$ organov mestnogo samoupravleniya» ot 09.02.2009 № 8-Ф3 [The Federal Law “On Ensuring Access to Information on the Activity of State and Local Authorities" of February 9, 2009 no. 8-FL]. Access from Reference Legal System "KonsultantPlyus".

6. Federalnyy zakon «Ob obespechenii dostupa $k$ informatsii o deyatelnosti sudov $v$ Rossiyskoy Federatsii» ot 22.12.2008 № 262-FZ (red. om 05.04.2016) [The Federal Law "On Ensuring Access to Information on the Activity of Courts in the Russian Federation" of December 22, 2008 no. 262-FL (ed. of April 5, 2016)]. Access from Reference Legal System "KonsultantPlyus".

7. Tikhomirov M.Yu., ed. Yuridicheskaya entsiklopediya [Legal Encyclopedia]. Moscow, Yurinformtsentr Publ., 2005. 972 p.

\section{Information About the Authors}

Yanina Ya. Keil, Candidate of Juridical Sciences, Assistant Professor, the Department of Civil Law Disciplines, Volgograd Institute of Management - the Branch of the Russian Presidential Academy of National Economy and Public Administration under the President of the Russian Federation, Gagarina St., 8,400131 Volgograd, Russian Federation, grajd@vags.ru.

Victoriya A. Usanova, Candidate of Juridical Sciences, Assistant Professor, the Department of Civil Law Disciplines, Volgograd Institute of Management - the Branch of the Russian Presidential Academy of National Economy and Public Administration under the President of the Russian Federation, Gagarina St., 8, 400131 Volgograd, Russian Federation, grajd@vags.ru.

\section{Информация об авторах}

Янина Яковлевна Кайль, кандидат юридических наук, доцент кафедры гражданско-правовых дисциплин, Волгоградский институт управления - филиал Российской академии народного хозяйства и государственной службы при Президенте РФ, ул. Гагарина, 8, 400131 г. Волгоград, Российская Федерация, grajd@vags.ru.

Виктория Александровна Усанова, кандидат юридических наук, доцент кафедры гражданско-правовых дисциплин, Волгоградский институт управления - филиал Российской академии народного хозяйства и государственной службы при Президенте РФ, ул. Гагарина, 8, 400131 г. Волгоград, Российская Федерация, grajd@vags.ru. 


\section{ТЕОРИЯ И ПРАКТИКА ГОСУДАРСТВЕННО-ПРАВОВОГО РАЗВИТИЯ}

\section{Informations sur les auteurs}

Janina Ya. Keil, Candidat des sciences juridiques, professeur adjoint des disciplines civiles et juridiques de l'Institut de l'Administration Publique de Volgograd - filiale de l'Académie Russe de l'Economie Nationale et de l'Administration Publique, 8, rue Gagarin, 400131, la ville de Volgograd, Russie, grajd@vags.ru.

Victoria A. Usanova, Candidat des sciences juridiques, professeur agrégé, Professeur adjoint des disciplines civiles et juridiques de l'Institut de l'Administration Publique de Volgograd - filiale de l'Académie Russe de l'Economie Nationale et de l'Administration Publique, 8, rue Gagarin, 400131, la ville de Volgograd, Russie, grajd@vags.ru. 